\title{
REVISÃo de LiTERATURA Atividade física sistematizada e sintomas de depressão na demência de Alzheimer: uma revisão sistemática
}

\author{
Systematized physical activity and depressive symptoms in Alzheimer's \\ dementia: a systematic review
}

Thays Martins Vital', Salma Stéphany Soleman Hernandez', Sebastião Gobbi', José Luiz Riani Costa', Florindo Stella $a^{1,2}$

\section{RESUMO}

Objetivo: Realizar uma revisão sistemática de artigos científicos que analisaram os efeitos da atividade física sistematizada nos sintomas de depressão de pacientes com demência de Alzheimer. Métodos: Foi realizada uma busca nas seguintes bases de dados: Web of Science, PubMed, PsycINFO, MedLine e Biological Abstracts, utilizando-se as seguintes palavras-chave: "Alzheimer dementia", "Alzheimer disease", "Alzheimer, physical activity", "physical exercise", "motor intervention", "physical therapy", "exercise", "aerobic", "strength", "fitness", "depression", "dysphoria", "depressive symptoms" e "depressive episodes". Além da busca nas bases de dados, foi realizada também uma busca manual nas listas de referências dos artigos selecionados. Resultados: Foram encontrados quatro estudos que preencheram todos os critérios de inclusão adotados para o presente trabalho. Dois estudos apresentaram reduções dos sintomas depressivos, e

\section{Palavras-chave}

Doença de Alzheimer, exercício físico, depressão.

\section{Keyword}

Alzheimer's disease, physical exercise, depression. outros dois não encontraram redução desses sintomas. Conclusão: Com a realização desta revisão sistemática, observou-se que não há consenso em relação aos benefícios da atividade física aos sintomas depressivos em pacientes com demência de Alzheimer.

\section{ABSTRACT}

Objective: To develop a systematic review of scientific articles that have been analyzed the effects of systematic physical activity in depression of patients with Alzheimer's disease. Methods: We performed a search in the following databases: Web of Science, PubMed, PsycINFO, MedLine and Biological Abstracts, we used the following keywords: "Alzheimer dementia", "Alzheimer disease", "Alzheimer's, physical activity", "physical exercise", "motor intervention", "physical therapy", "exercise", "aerobic", "strength", "fitness", "depression", "dysphoria", "depressive symptoms" and "depressive episodes", and also a manual search in references of the selected articles. Results: Have been found four studies that met all criteria of this study. Two studies presented reductions in depressive symptoms, and two others no reduction of these symptoms. Conclusion: In conducting this systematic review, it was observed that there is not consensus regarding the benefits of physical activity on depressive symptoms in patients with Alzheimer dementia.

1 Universidade Estadual Paulista (Unesp), Instituto de Biociências (Campus de Rio Claro), Departamento de Educação Física, Laboratório de Atividade Física e Envelhecimento. 


\section{INTRODUÇÃO}

Os sintomas neuropsiquiátricos como agitação, disforia, apatia, irritabilidade, comportamento motor aberrante, delírios, alucinações, desinibição, depressão e distúrbios de sono são frequentemente observados em pacientes com demência e tendem a aumentar conforme avança a doença $a^{1,2}$. Tais sintomas estão diretamente associados com aumento da sobrecarga nos cuidadores e podem aumentar o risco de institucionalização precoce do paciente ${ }^{3-5}$.

Acredita-se que sintomas como humor depressivo clinicamente significativo, diminuição de prazer aos contatos sociais ou usuais, isolamento ou retraimento social, alteração do apetite e do ciclo vigília-sono, alterações psicomotoras, irritabilidade, fadiga e sentimento de inutilidade, desesperança ou culpa excessiva e inapropriada, pensamentos recorrentes de morte e ideação suicida estejam presentes, associados ou isolados, em pacientes com demência de Alzheimer (DA), o que pode resultar em incapacidade física e morte prematura ${ }^{6,7}$.

A frequência de depressão maior em pacientes com DA varia de $5 \%$ a $23 \%$ como elucida a literatura ${ }^{8-12}$. Pacientes com DA deprimidos têm maior deficiência na execução das atividades de vida diária9,13 e maior probabilidade de episódios de agitação, além de apresentarem mais riscos de institucionalização e aumento do estresse no cuidador ${ }^{14,15}$.

A depressão no idoso é considerada como um processo multifatorial, ou seja, desencadeada por diferentes aspectos: sociais, psíquicos, cognitivos e físicos ${ }^{16}$. Dessa forma, tornam-se difíceis o diagnóstico e o tratamento desses pacientes, sendo viável a associação de diferentes tratamentos de caráter farmacológico e não farmacológico.

A atividade física regular deve ser considerada como uma alternativa não farmacológica de tratamento do transtorno depressivo, por representar baixo custo econômico, ser acessível e prevenir o declínio funcional do idoso. O exercício físico apresenta contribuições importantes, sobretudo quando associado ao tratamento psicofarmacológico da depressão, auxiliando na recuperação da autoestima e da autoconfiança ${ }^{17}$.

Existem estudos que demonstram que o exercício físico reduz os sintomas depressivos de idosos preservados cognitivamente ${ }^{18-26}$. Porém, sabe-se ainda muito pouco sobre os efeitos do exercício físico nos sintomas depressivos em idosos com DA. Além de tudo, é importante investigar qual tipo de atividade é mais eficiente e qual a melhor intensidade e frequência para promover melhora na saúde dessa população.

Dois estudos realizados por Arkin ${ }^{27,28}$ aplicaram um protocolo de intervenção motora combinada que abordou diferentes componentes da capacidade funcional. O primeiro estudo teve duração de quatro anos e o segundo foi realizado durante um ano. Ambas as intervenções foram eficientes para reduzir os escores da Geriatric Depression Scale (GDS) ${ }^{29}$.
Destaca-se aqui a importância da atividade física na redução desses sintomas. Porém, ambos deixam lacunas em relação à validade das medidas avaliadas. Essas limitações foram geradas em virtude da ausência de grupo-controle. Evidencia-se, assim, a importância de que sejam realizados estudos controlados, a fim de verificar os reais efeitos da atividade física nos pacientes com DA.

Diante dessa realidade, o objetivo deste estudo foi buscar e analisar, por meio de uma revisão sistemática, artigos que verifiquem os efeitos da atividade física sistematizada nos sintomas depressivos de pacientes com DA.

\section{MÉTODOS}

O processo metodológico deste estudo consistiu em uma revisão sistemática da literatura, orientada pela busca bibliográfica nas seguintes bases de dados: Web of Science, PubMed, PsycINFO, MedLine e Biological Abstracts, de 1990 a março de 2009. Essas bases foram escolhidas por abordarem especificamente assuntos que envolvessem a área da saúde. Os operadores booleanos e as palavras-chave utilizados foram: Alzheimer dementia OR Alzheimer disease OR Alzheimer AND physical activity OR physical exercise OR motor intervention OR physical therapy OR exercise OR aerobic OR strength OR fitness AND depression OR dysphoria OR depressive symptoms OR depressive episodes. Além da busca nas bases de dados, realizou-se uma busca manual nas listas de referências dos artigos selecionados. A busca dos artigos iniciou-se em março de 2009, sendo adotados os seguintes critérios de inclusão: (1) diagnóstico da doença de Alzheimer segundo os critérios oficiais de órgãos internacionais (CID, DSM, NINCDS/ADRDA); (2) estudos longitudinais controlados; (3) estudos que realizaram como protocolo de intervenção, atividade física sistematizada; (4) estudos que apresentaram como variável dependente sintomas de depressão. Aqueles artigos que não atenderam a esses critérios de inclusão foram excluídos do estudo.

\section{RESULTADOS}

A busca bibliográfica resultou em 272 artigos. Em uma primeira análise, por meio da leitura do título, verificou-se que 221 artigos não se relacionavam com o tema proposto, restando 51 artigos. Logo após realizou-se uma leitura dos resumos desses artigos selecionados e observou-se que 34 não se relacionavam ao tema, restando, assim, 17 artigos. Por meio de uma leitura desses artigos na íntegra, foram selecionados 4 artigos que se enquadravam no objetivo do estudo. Os 13 artigos excluídos nessa fase final não se enquadravam nos critérios de inclusão do estudo: 4 artigos não tinham intervenção motora; 3 artigos eram revisões de literatura; 
1 artigo não possuía diagnóstico de DA; 2 artigos não se referiam a sintomas de depressão como variável dependente; 1 artigo apresentava amostra heterogênea; 2 artigos não eram controlados.

A seguir, a descrição dos quatro estudos selecionados nesta revisão sistemática:

1) O estudo de Williams e Tappen ${ }^{30}$ adotou três tipos de tratamento, no qual os participantes foram randomizados (grupo 1: exercícios de força, equilíbrio, flexibilidade e aeróbico; grupo 2: caminhada individual; grupo 3: controle). Para os grupos com intervenção, as sessões aumentavam gradativamente até chegar a 30 minutos de duração. A intervenção teve duração de 16 semanas, 5 vezes por semana. Participaram desse estudo 45 pacientes institucionalizados (média de idade de 87,9 $\pm 5,9$ anos), que foram acompanhados durante as sessões por enfermeiros ou estudantes de fisioterapia que estavam treinados e supervisionados durante toda a intervenção. Após 16 semanas de tratamento, os autores encontraram reduções significativas nos sintomas depressivos, avaliados pela Cornell Scale for Depression in Dementia $(C S D D)^{31}$, em todos os grupos submetidos ao tratamento, com evidência de maiores benefícios nos grupos que realizaram a intervenção motora.

2) Steinberg et al. ${ }^{32}$ realizaram um estudo durante 12 semanas, que consistiu em dois tipos de tratamentos: grupo 1: exercícios aeróbicos, força, equilíbrio e flexibilidade; grupo 2: controle. Participaram desse estudo 27 pacientes institucionalizados, randomizados em grupo-controle (média de idade de $74 \pm 8,1$ anos) e intervenção (média de idade de 76,5 $\pm 3,9$ anos). Realizaram-se as avaliações no momento anterior e no momento final da $6^{\mathrm{a}}$ e $12^{\mathrm{a}}$ semana, para ambos os grupos. No final das 12 semanas, não foram encontradas diferenças significativas entre os grupos nos sintomas depressivos avaliados pela CSDD ${ }^{31}$ e pelo Neuropsychiatric Inventory (NPI) - domínio: depressão ${ }^{33}$. Esse estudo não especificou a frequência semanal do programa de exercícios físicos nem a frequência em que eram transmitidas as informações de segurança doméstica.

3) Do estudo de Rolland et al.34 participaram 134 idosos institucionalizados. Eles foram divididos de forma randomizada em grupo-treinamento (67 participantes, com média de idade de 82,8 \pm 7,8 anos) e grupo-controle (67 participantes, com média de idade de 83,1 \pm 7 anos). Adotou-se também como critério de inclusão um valor menor que 25 pontos no Miniexame do Estado Menta| ${ }^{35}$. O protocolo de intervenção consistiu em exercícios aeróbicos, força, equilíbrio e flexibilidade. Os exercícios aeróbicos preencheram a maior parte da aula e a intensidade dos exercícios aumentava gradualmente após o primeiro mês de atividade. O programa de intervenção motora teve duração de um ano, no qual era realizado duas vezes na semana. O grupo-controle participou somente da rotina médica. As avaliações foram realizadas no momento anterior, após seis meses e no final do treinamento para ambos os grupos. Somente 110 participantes completaram o estudo. Os autores não encontraram diferença estatisticamente significante em nenhum dos momentos nos sintomas depressivos avaliados pela Montgomery-Asberg Depression Rating Scale (MADRS) ${ }^{36}$ entre o grupo-treinamento e o grupo-controle. Além disso, a porcentagem de pacientes com depressão também não reduziu em ambos os grupos.

4) Do estudo realizado por Teri et al. ${ }^{37}$ participaram 153 idosos com DA, divididos de maneira randomizada em grupo-intervenção, com 76 participantes (média de idade de 78 \pm 6 anos), e grupo-controle, que realizava apenas sua rotina médica, com 77 participantes (média de idade de $78 \pm$ 8 anos). $O$ tratamento aplicado teve duração de três meses, realizado na casa do paciente. Nas três primeiras semanas foram realizadas duas sessões, seguidas por uma sessão semanal durante quatro semanas, e logo em seguida realizavam apenas uma sessão a cada 15 dias, até o final do tratamento. A sequência de exercícios consistiu em atividades aeróbicas, força, equilíbrio e flexibilidade. Os exercícios tinham duração de 30 minutos, com variação da intensidade de leve a moderada. Após os três meses de tratamento, foram realizadas três visitas para que os cuidadores respondessem a questões sobre os efeitos do tratamento. Essas visitas foram realizadas até se completarem dois anos após a data inicial do início do tratamento. Concluíram o estudo, após esses dois anos, 89 idosos. Com análise dos dados, os autores encontraram meIhora significativa nos sintomas depressivos avaliados pela $\mathrm{CSDD}^{31}$ e pela Escala de Depressão de Hamilton (HAD) ${ }^{38,39}$ no grupo que realizou o tratamento em relação ao grupo-controle, após os três meses de tratamento, e mantiveram essa melhora após dois anos do início da intervenção. Entretanto, a HAD só foi aplicada quando o idoso apresentava escore acima de 6 pontos na CSDD.

A tabela 1 apresenta as características e os resultados dos estudos revisados.

\section{DISCUSSÃO}

Ao se analisarem os 17 artigos selecionados inicialmente, quatro artigos foram incluídos na análise final deste estudo. Desses quatro estudos, dois apresentaram reduções dos sintomas depressivos ${ }^{30,37}$ e dois não encontraram reduções desses sintomas ${ }^{32,34}$.

Em relação à metodologia adotada nos trabalhos selecionados, alguns aspectos importantes geram limitações que interferem nos resultados encontrados, tais como: aplicação de atividade física generalizada, uso de amostras reduzidas e curto período de intervenção.

Os estudos de Williams e Tappen ${ }^{30}$ e Teri et al..$^{37}$ encontraram redução dos sintomas depressivos nos grupos que realizavam atividade física. No estudo de Williams e Tappen ${ }^{30}$ existiam dois grupos com intervenção motora e um grupo-controle. 
Tabela 1. Características dos estudos que examinaram os efeitos da atividade física nos sintomas de depressão em idosos com demência de Alzheimer

\begin{tabular}{|c|c|c|c|c|c|}
\hline \multirow{2}{*}{ Autor } & \multicolumn{2}{|c|}{ Amostra } & \multirow[t]{2}{*}{ Avaliação } & \multirow{2}{*}{$\begin{array}{l}\text { Intervenção } \\
\text { Tarefa motora }\end{array}$} & \multirow[t]{2}{*}{ Resultados } \\
\hline & Tamanho & Caracterização & & & \\
\hline 1. Williams e Tappen ${ }^{30}(2008)$ & $\begin{array}{l}\mathrm{n}=45 \text { pacientes } \\
\text { institucionalizados }\end{array}$ & $\begin{array}{l}\text { Alzheimer provável } \\
\text { (critério: NINCDS - } \\
\text { ADRDA) }\end{array}$ & $\begin{array}{l}\text { CSDD (Cornell Scale for } \\
\text { Depression in Dementia) }\end{array}$ & $\begin{array}{l}\text { Grupo I: } 10 \text { minutos de } \\
\text { exercícios de força, equilíbrio } \\
\text { e flexibilidade; caminhada } \\
\text { que chegou até } 20 \text { minutos; } \\
\text { grupo Il: caminhada; a duração } \\
\text { chegou a } 30 \text { minutos; grupo III: } \\
\text { grupo de conversação, sem } \\
\text { intervenção motora }\end{array}$ & $\begin{array}{l}\text { Todos os grupos apresentaram } \\
\text { reduções significativas nos sintomas } \\
\text { depressivos }\end{array}$ \\
\hline 2. Steinberg et al.32 (2009) & $\begin{array}{l}\mathrm{n}=27 \text { pacientes } \\
\text { institucionalizados }\end{array}$ & $\begin{array}{l}\text { Alzheimer provável } \\
\text { (critério: NINCDS - } \\
\text { ADRDA) }\end{array}$ & $\begin{array}{l}\text { CSDD (Cornell Scale for } \\
\text { Depression in Dementia); } \\
\text { NPI (Neuropsychiatric } \\
\text { Inventory - domain: } \\
\text { depression) }\end{array}$ & $\begin{array}{l}\text { Grupo I: exercício aeróbico: } \\
\text { caminhada, podendo substituir } \\
\text { a atividade física por outra } \\
\text { uma vez na semana, mas com a } \\
\text { mesma intensidade; exercício } \\
\text { de força; exercícios de equilí- } \\
\text { brio e flexibilidade. Grupo Il: } \\
\text { participaram de um programa } \\
\text { de segurança em casa, no qual } \\
\text { recebiam instruções de como } \\
\text { adaptar melhor a casa para } 0 \\
\text { paciente com DA }\end{array}$ & $\begin{array}{l}\text { Não foram encontradas diferenças } \\
\text { significativas em nenhuma das escalas } \\
\text { utilizadas }\end{array}$ \\
\hline 3. Roland et al. ${ }^{34}$ (2007) & $\begin{array}{l}\mathrm{n}=134 \text { pacientes } \\
\text { institucionalizados }\end{array}$ & $\begin{array}{l}\text { Alzheimer provável } \\
\text { (critério: NINCDS - } \\
\text { ADRDA) } \\
\text { MEEM } \leq 25 \text { pts }\end{array}$ & $\begin{array}{l}\text { MADRS (Montgomery- } \\
\text { Asberg Depression } \\
\text { Rating Scale) }\end{array}$ & $\begin{array}{l}\text { Grupo I: exercícios aeróbicos, } \\
\text { força, equilíbrio e flexibilidade. } \\
\text { Grupo Il: sem intervenção } \\
\text { motora }\end{array}$ & $\begin{array}{l}\text { Não foram encontradas melhoras sig- } \\
\text { nificativas nos sintomas depressivos. } \\
\text { A proporção de idosos com depressão } \\
\text { não mudou significativamente }\end{array}$ \\
\hline 4. Teri et al. ${ }^{37}$ (2003) & $\begin{array}{l}\mathrm{n}=153 \text { pacientes de } \\
\text { uma Associação de } \\
\text { pacientes com DA }\end{array}$ & $\begin{array}{l}\text { Alzheimer provável } \\
\text { (critério: NINCDS - } \\
\text { ADRDA) }\end{array}$ & $\begin{array}{l}\text { CSDD (Cornel Scale for } \\
\text { Depression in Dementia); } \\
\text { HAD (Hamilton Depres- } \\
\text { sion Rating Scale) }\end{array}$ & $\begin{array}{l}\text { Grupo I: exercícios aeróbicos, } \\
\text { força, equilíbrio e flexibilidade. } \\
\text { Grupo Il: sem intervenção } \\
\text { motora }\end{array}$ & $\begin{array}{l}\text { Redução dos sintomas depressivos } \\
\text { no grupo que realizou a intervenção } \\
\text { motora }\end{array}$ \\
\hline
\end{tabular}

Os autores mostraram com esse estudo que todos os três grupos reduziram os sintomas depressivos após o período de intervenção. Porém, a redução desses sintomas foi maior nos dois grupos que praticavam atividade física, destacando, assim, a importância da associação da atividade física sistematizada e regular com o tratamento farmacológico desses pacientes com DA. Já o estudo de Teri et al. ${ }^{37}$ possuía dois grupos: controle e intervenção. Os autores encontraram redução dos sintomas depressivos no grupo que realizou a intervenção motora. Entretanto, ela foi realizada na casa do próprio paciente, criando limitações em relação à sobrecarga e ao controle da atividade proposta.

Outros dois estudos, realizados por Steinberg et al. ${ }^{32} \mathrm{e}$ Rolland et al. ${ }^{34}$, não encontraram melhoras significativas nos sintomas depressivos dos grupos submetidos à atividade física. No primeiro estudo, os autores aplicaram um protocolo de atividade física generalizada durante 12 semanas, e a falta de resultados significativos foi atribuída ao tamanho reduzido da amostra. No estudo de Rolland et al. ${ }^{34}$, também foi aplicado um programa de atividade física generalizada durante um ano, e os autores enfatizavam que a duração da intervenção não foi suficiente para reduzir os sintomas depressivos do grupo que realizou a atividade física. Além disso, observa-se que em ambos os estudos ${ }^{32,34}$ os autores também não encontraram melhora significativa nos testes motores desses pacientes. Dessa forma, parece que o tipo de treinamento aplicado não foi suficiente para promover mudanças fisiológicas, justificando, assim, talvez, a falta de resultados positivos na parte comportamental, nesse caso os sintomas depressivos.

No estudo, Steinberg et al. ${ }^{32}$ não citavam o nível de estagiamento da doença, gerando, assim, limitações, visto que ainda não existe um consenso na literatura em relação ao nível de estagiamento da demência e da depressão nesses pacientes ${ }^{40,41}$. Em razão da falta desse consenso, é difícil inferir se pacientes com menores ou maiores níveis de gravidade da DA podem apresentar mais sintomas depressivos.

Destaca-se, também, que somente dois estudos ${ }^{30,34}$ cita- $^{-}$ ram o uso de antidepressivos. No estudo de Williams e Tappen $^{30}$, foi verificado que a quantidade de antidepressivos não reduziu após o período de intervenção motora. Já no estudo de Rolland et al. ${ }^{34}$, as informações do uso de antidepressivos só foram coletadas no momento pré-intervenção. É necessária a realização de novos estudos que acompanhem melhor o uso de antidepressivos, que podem ser fortes indicadores na redução dos sintomas de depressão.

Os protocolos de atividade física aplicados à maioria dos estudos foram de caráter geral, ou seja, as atividades 
desenvolvidas abordavam os componentes da capacidade funcional, tais como capacidade aeróbica, força, equilíbrio e flexibilidade. Três estudos ${ }^{32,34,37}$ possuíam dois grupos em avaliação; o primeiro grupo realizou como intervenção exercícios de caráter geral e o outro grupo foi considerado rotina médica. Já no estudo de Steinberg et al. ${ }^{32}$, realizavam com o grupo-controle uma avaliação da segurança doméstica desses pacientes. A presença de um grupo-controle representa maior qualidade para os resultados encontrados, afinal ainda não existe um consenso na literatura que afirme que atividade física é benéfica na redução dos sintomas depressivos em pacientes com DA.

Os estudos citados anteriormente, que abordavam atividade física de forma mais ampla, sem trabalhar especificamente com algum componente da capacidade funcional, impedem que se conclua qual o tipo de atividade é mais benéfico para pacientes com DA.

Eggermont et al. ${ }^{42}$, em uma revisão, apontam que o exercício físico não é necessariamente benéfico para todos os pacientes com demência. Os efeitos positivos do exercício no aumento da perfusão cerebral dependem da condição cardíaca do paciente. Sendo assim, deve ser realizada uma avaliação mais profunda, verificando principalmente as suas condições cardíacas.

Os estudos de Steinberg et al. ${ }^{32}$ e Rolland et al. ${ }^{34}$ apresentam limitações em relação à metodologia do treinamento aplicado, pois não descrevem como era feita a sobrecarga do treinamento, somente citam que a intensidade era aumentada a cada aula.

Segundo Stella et al.43, a atividade física regular deve ser considerada como uma alternativa não farmacológica do tratamento do transtorno depressivo. O autor ainda complementa que, do ponto de vista mental, a atividade física, sobretudo quando praticada em grupo, eleva a autoestima do idoso e contribui para a implementação das relações psicossociais e para o reequilíbrio emocional. Capacidade de atenção concentrada, memória de curto prazo e desempenho dos processos executivos (planejamento de ações sequenciais logicamente estruturadas e capacidade de autocorreção das ações) constituem funções cognitivas imprescindíveis na vida cotidiana, que são estimuladas durante a prática de exercícios bem planejados.

Somente no estudo de Williams e Tappen ${ }^{30}$ foram utilizados dois grupos de intervenção, um grupo apresentava o caráter de atividade física generalizada e outro grupo só realizou atividade aeróbica, mais especificamente caminhada. A redução dos sintomas depressivos foi mais expressiva nos pacientes que realizaram esse tipo mais específico de atividade. Em estudo de revisão, Deslandes et al..$^{44}$ evidenciam mecanismos envolvidos pela prática da atividade física, como os aspectos afetivos, comportamentais, psicossociais e motores que corroboram os achados de Stella et all. ${ }^{43}$. Acrescentam, ainda, uma hipótese neurofisiológica dessa prática como um assunto escasso e promissor para o tratamento/prevenção dos transtornos depressivos. Essa hipótese, por sua vez, aborda mecanismos como a hormese, produção de espécies reativas ao oxigênio, diminuindo ações antioxidantes e que podem aumentar a imunidade biológica, aumento da produção do fator neurotrófico derivado do cérebro e do fator de crescimento ligado à insulina, que promovem neurogênese e angiogênese, mediando melhoras comportamentais, além de aumentar a produção e a alteração nos receptores de neurotransmissores como serotonina, dopamina, acetilcolina e norepinefrina, resultando em aumento da perfusão cerebral.

A falta de estudos nessa temática abordada resulta em alguns questionamentos como qual a melhor atividade física, duração e frequência para essa população com DA. Desses quatro trabalhos estudados, observa-se que a frequência semanal variou de duas a cinco vezes na semana, a duração das sessões variaram de 30 minutos a 1 hora e a duração das intervenções variaram de três meses a um ano. Sendo assim, por causa da grande variabilidade de protocolos de treinamento, novos estudos são necessários para que se crie um consenso de qual é o melhor protocolo de treinamento para atenuar os sintomas depressivos em pacientes com DA.

Outra questão importante quando são realizados estudos com depressão em idosos é o fato de acompanhar esse grupo após o período de intervenção aplicada, mesmo depois da remissão da doença, pois, uma vez instalado um quadro depressivo, o paciente se torna mais suscetível a recaídas. Dentre os estudos analisados, somente um estudo ${ }^{37}$ apresentou essa característica, com acompanhamento dos grupos estudados até completar dois anos após o período de intervenção. Nesse estudo, eles observavam que, mesmo depois do término da intervenção, os indivíduos não aumentavam os sintomas depressivos, apenas mantiveram o quadro que já estava instalado. Vale ressaltar que, segundo alguns autores ${ }^{45-47}$, não se pode descartar a hipótese de que a depressão pode ser um fator de risco para demência, ou mesmo preceder o surgimento da DA. Dessa forma, o tratamento da depressão em pacientes com DA pode melhorar substancialmente a cognição ${ }^{48}$.

O tamanho reduzido da amostra pode interferir nos resultados encontrados, ou seja, amostras que não representem de forma significativa a população estudada. A maior amostra utilizada nos estudos analisados foi de 153 sujeitos e a menor, de 30 sujeitos. Em todos os estudos a amostra foi heterogênea, não especificando o número exato de homens e mulheres em cada estudo. A maioria dos sujeitos é institucionalizada ${ }^{30,34}$ ou de grupos da comunidade que atendem esses pacientes com DA ${ }^{32,37}$. Canineu ${ }^{49}$ afirma que os pacientes com demência apresentam maior dependência, de acordo com a evolução do quadro clínico, levando a maior risco de institucionalização deles.

Em relação às escalas aplicadas, houve maior utilização da Cornell Scale for Depression in Dementia (CSDD) ${ }^{31}$, que é 
uma escala validada e específica para esse tipo de população e incorpora informações adquiridas com o cuidador do paciente com DA. Outras escalas como Neuropsychiatric Inventory (NPI) - domínio: depressão ${ }^{33}$, Montgomery-Asberg Depression Rating Scale (MADRS) $)^{36}$ e Hamilton Depression Rating Scale (HAD) ${ }^{38,39}$ também foram utilizadas. A maioria das escalas de depressão existente baseia-se em informações fornecidas pelos pacientes, que preenchem um questionário ou são entrevistados por um profissional de saúde devidamente treinado. Esse método torna difícil a avaliação de pacientes com demência, já que suas falhas de concentração, memória e julgamento podem prejudicar a interpretação de suas respostas ${ }^{50}$. Ressalta-se a importância da elaboração e utilização de escalas que são específicas para avaliar a depressão em idosos, e mais especificamente para idosos com demência. Entretanto, o estado cognitivo do paciente pode afetar suas respostas, resultando em falsas interpretações, e nesses casos é ideal que se acrescentem também as respostas do cuidador desse paciente ${ }^{51}$. Sendo assim, ainda existem algumas limitações nos estudos analisados, visto que o mais viável seria que o diagnóstico de depressão fosse realizado por um clínico especializado na área, reduzindo, assim, possíveis erros de diagnóstico.

A randomização ocorreu nos quatro estudos selecionados, porém aconteceram de diferentes formas. Williams e Tappen ${ }^{30}$ e Rolland et al..$^{34}$ selecionaram a amostra por meio de sorteio. Já nos estudos de Teri et al..$^{37}$ foi utilizado um programa de computador para randomização da amostra, porém não se especificou quais os critérios de seleção adotados por esse programa. E, por último, outro estudo de Steinberg et al..$^{32}$ escolheu randomização estratificada, na qual os sujeitos foram separados para que ambos os grupos apresentassem a mesma quantidade de homens e mulheres e de indivíduos acima e abaixo de 75 anos. Dessa forma, parece que esse tipo de randomização, dentre as verificadas nesses quatro estudos, conseguiu homogeneizar melhor os sujeitos alocados em cada grupo, reduzindo, assim, algumas variáveis confundidoras.

Um último aspecto a ser analisado nesta revisão refere-se a um fator confundidor quando se tratam de atividade física e sintomas de depressão, que é o fator social. Afinal, é muito difícil separar esses dois aspectos, pois a maioria dos estudos não consegue controlar até que ponto o fato de o paciente sair da sua rotina e estar envolvido em um grupo realizando a intervenção motora pode gerar bem-estar psicológico, físico e social. Esse fato é explicado por Peluso e Andrade ${ }^{52}$, por meio da hipótese da interação social: que é comumente inerente, inseparável da atividade física, assim como o suporte mútuo que aparece entre indivíduos envolvidos no exercício; jogar tem um importante papel nos efeitos do exercício na saúde mental.

Como se pode observar, ainda não existe um consenso em relação a qual é a melhor modalidade de atividade físi- ca para redução dos sintomas depressivos, pois, dos quatro estudos analisados, dois apresentaram efeitos positivos, ou seja, reduziram os sintomas depressivos, e os outros dois não reduziram.

Diante disso, são necessários novos estudos que descubram os efeitos da atividade física para essa população com DA. Além disso, é necessário criar estratégias que atenuem os distúrbios neuropsiquiátricos, mais especificamente os sintomas de depressão, que acomete a grande maioria dessa população. Verifica-se, assim, o papel da atividade física como intervenção não farmacológica no auxílio ao tratamento da DA, proporcionando para esses pacientes e cuidadores meIhor qualidade de vida e bem-estar.

\section{CONCLUSÃO}

Este trabalho de revisão sistemática mostrou que ainda não há um consenso em relação aos benefícios do exercício para essa população. Além disso, existem controvérsias em relação ao melhor tipo de exercício, intensidade e duração para que ele possa reduzir os sintomas depressivos em pacientes com DA. Diante dessa realidade, fica a necessidade da realização de novos estudos controlados, para que se possa atingir um consenso em relação a qual o melhor tipo de atividade física para o tratamento dos sintomas depressivos nos pacientes com DA.

\section{REFERÊNCIAS}

1. Schulz R, O'Brien AT, Bookwala J, Fleissner K. Psychiatric and physical morbidity effects of dementia caregiving. Gerontologist. 1995;35:771-91.

2. Lyketsos CG, Steinberg M, Tschanz JT, Norton MC, Steffens DC, Breitner JCS. Mental and behavioral disturbances in dementia: findings from the cachas county study on memory and aging. Am J Psychiatry. 2000;157:708-14.

3. Tan $L L$, Wong $H B$, Allen $H$. The impact of neuropsychiatric symptoms of dementia on distress in family and professional caregivers in Singapore. Int Psychogeriatr. 2005;17:253-63.

4. Magni R, Binetti G, Bianchetti A, Trabucchi M. Risk of mortality and institutionalization in demented patients with delusion. J Geriatr Psychiatry Neurol. 1996;9:123-6.

5. Scarmeas N, Brandt J, Albert M, Hadjigeorgiou G, Papadimitriou A, Dubois B. Delusions and hallucinations are associated with worse outcome in Alzheimer disease. Arch Neurol. 2005;62:1601-8.

6. Olin JT, Schneider LS, Kats IR, Meyers BS, Alexopoulos GS, Breitener JC, et al. Provisional diagnostic criteria for depression of Alzheimer disease. Am J Geriatric Psychiatr. 2002;10:125-8

7. Snowdon J. How high is the prevalence of depression in old age? Rev Bras Psiquiatr. 2002;24:42-7.

8. Lopez OL, Becker JT, Sweet RA, Klunk W, Kaufer DI, Saxton J, et al. Psychiatric symptoms vary with the severity of dementia in probable Alzheimer's disease. J Neuropsychiatry Clin Neurosci. 2003;15(3):346-53.

9. Lyketsos CG, Baker L, Warren A, Steele CD, Brandt J, Steinberg M. Major and minor depression in Alzheimer's disease: prevalence and impact. J Neuropsychiatry Clin Neurosci. 1997;9:556-61.

10. Starkstein SE, Chemerinski E, Sabe L, Kuzis G, Petracca G, Tesón A, et al. Prospective longitudinal study of depression and anosognosia in Alzheimer's disease. Br J Psychiatry. 1997;171:47-52. 
11. Butt ZA, Strauss M. Relationship of family and personal history to the occurrence of depression in persons with Alzheimer's disease. Am J Geriatr Psychiatry. 2001;9(3):249-54.

12. Regan C, Katona C, Walker Z, Livingston G. Relationship of exercise and other risk factors to depression of Alzheimer's disease: the LASER-AD study. Int I Geriatr Psychiatry. 2005;20:261-8.

13. Lyketsos CG, Steele C, Galik E, Rosenblatt A, Steinberg M, Warren A, et al. Physical aggression in dementia patients and its relationship to depression. Am J Psychiatry. 1999;156:66-71.

14. Gonzales-Salvator T, Aragano C, Lyketsos CG, Barba AC. The stress and psychological morbidity of the Alzheimer patient caregiver. Int J Geriatr Psychiatry. 1999;14:701-10.

15. Hoch CC, Reynolds CF, Buysse DJ. Two-year survival in patients with mixed symptoms of depression and cognitive impairment: comparison with major depression and primary degenerative dementia. Am J Geriatr Psychiatry. 1993;1:59-66.

16. Bottino CMC. The challenge of treating of depression in the elderly. Int Clin Psychopharmacol. 2003;18(Suppl 1):S39-45.

17. Guimarães JMN, Caldas CP. A influência da atividade física nos quadros depressivos de pessoas idosas: uma revisão sistemática. Rev Bras Epidemiol. 2006;9(4):481-92.

18. Blumenthal JA, Babyak MA, Moore K, Craighead WE, Herman S, Doraiswamy M, et al. Effects of exercise training on older patients with major depression. Arch Intern Med. 1999;159:2349-56.

19. MCNeil JK, LeBlanc EM, Joyner M. The effect of exercise on depressive symptoms in the moderately depressed elderly. Psychol Aging. 1991;6(3):487-8.

20. Kohut ML, Lee W, Martin A, Arnston B, Russell DW, Ekkekakis P, et al. The exercise-induced enhancement of influenza immunity ismediated in part by improvements in psychosocial factors in older adults. Brain Behav Immun. 2005:19(4):357-66.

21. Singh NA, Stavrinos TM, Scarbek Y, Galambos G, Liber C, Fiatarone Singh MA. A randomized controlled trial of high versus general practitioner care for clinical depression in older adults. J Gerontol A Biol Sci Med Sci. 2005;60(6):768-76.

22. Motl RW, Konopack JF, MCAuley E, Elavsky S, Jerome GJ, Marquez DX. Depressive symptoms among older adults: Iong-term reduction after physical activity intervention. J Behav Med. 2005:28(4):385-94.

23. Castro CM, Wilcox S, O'Sullivan P, Bauman K, King AC. An exercise program for women who are caring for relatives with dementia. Psychosom Med. 2002;64(3):458-68.

24. Antunes HK, Stella SG, Santos RF, Bueno OF, de Mello MT. Depression, anxiety and quality of life scores in seniors after an endurance exercise program. Rev Bras Psiquiatr. 2005;27(4):266-71.

25. Lai SM, Studenski S, Richards L, Perera S, Reker D, Rigler S, et al. Therapeutic exercise and depressive symptoms after stroke. J Am Geriatr Soc. 2006;54(2):240-7.

26. Mather AS, Rodriguez C, Guthrie MF, McHarg AM, Reid IC, McMurdo ME. Effects of exercise on depressive symptoms in older adults with poorly responsive depressive disorder: randomized controlled trial. Br J Psychiatry. 2002;180:411-5.

27. Arkin S. Elder Rehab: A Student-Supervised Exercise Program for Alzheimer's Patient. The Gerontologist. 1999;39(6):729-35.

28. Arkin S. Student-led exercise sessions yield significant fitness gains for Alzheimer's patients. Am J Alzheimers Dis Other Demen. 2003;18(3):159-70.

29. Yesavage JA, Brink TL, Rose TL, Lum 0, Huang V, Adey M, et al. Development and validation of a geriatric depression screening scale: a preliminary report. J Psychiat Res. 1983;17:37-49.

30. Williams CL, Tappen RM. Exercise for depressed older adults with Alzheimer disease. Aging Ment Health. 2008;12(1):72-80.

31. Alexopoulos GS, Abrams RC, Young RC, Shamoian CA. Cornell Scale for Depression in Dementia. Biol Psychiatry. 1988;23:271-84.
32. Steinberg M, Leoutsakos JS, Podewils LJ, Lyketsos CG. Evaluation of a home-based exercise program in the treatment of Alzheimer's disease: The Maximizing Independence in Dementia (MIND) study. Int J Geriatr Psychiatry. 2009;24(7):680-5.

33. Cummings JL, Mega M, Gray K, Rosenberg-Thompson S, Carusi DA, Gornbein J. The Neuropsychiatric Inventory: Comprehensive assessment of psychopathology in dementia. Neurology. 1994;44:2308-14.

34. Rolland Y, Pillard F, Klapouszczak A, Reynish E, Thomas D, Andrieu S, et al. Exercise Program for Nursing Home Residents with Alzheimer's Disease: A 1-Year Randomized, Controlled Trial. JAGS. 2007;55:158-65.

35. Folstein MF. Mini Mental State. A practical method for grading the cognitive state of patients for the clinicians. J Psychiatr Res. 1975;12:189-98.

36. Muller-Thomsen T, Arlt S, Mann U, Mass R, Ganzer S. Detecting depression in Alzheimer's disease: evaluation of four different scales. Arch Clin Neuropsychol. 2005;20:271- 6 .

37. Teri L, Gibbons LE, McCurry SM, Logsdon RG, Buchner DM, Barlow WE, et al. Exercise plus behavioral management in patients with Alzheimer disease: a randomized controlled trial. JAMA. 2003;290:2015-22.

38. Hamilton M. A rating scale for depression. J Neurol Neurosurg Psychiatry. 1960;23:56-62.

39. Hamilton M. Development of a rating scale for primary depressive illness. Br J Soc Clin Psychol. 1967;6:278-96.

40. Fischer P, Simanyi M, Danielczyk W. Depression in dementia of the Alzheimer type and multi-infarct dementia. Am J Psychiatry. 1990;147:1484-7.

41. Rovner BW, Broadhead J, Spencer M. Depression in Alzheimer's disease. Am J Psychiatry. 1989;146:350-3.

42. Eggermont L, Swaab D, Luiten P, Scherder E. Exercise, cognition and Alzheimer's disease: more is not necessarily better. Neurosci Biobehav Rev. 2006;30:652-575.

43. Stella F, Gobbi S, Corazza DI, Costa JLR. Depressão no idoso: diagnóstico, tratamento e benefícios da atividade física. Motriz. 2002;8(3):91-8.

44. Deslandes A, Moraes H, Ferreira C, Veiga H, Silveira H, Mouta R, et al. Exercise and mental health: many reasons to move. 2009;59:191.

45. Speck CE, Kukull WA, Brenner DE, Bowen JD, McCormick WC, Teri L, et al. History of depression as a risk factor for Alzheimer's disease. Epidemiology. 1995;6:366-9.

46. Ownby RL, Crocco E, Acevedo A, John V, Loewenstein D. Depression and risk for Alzheimer disease: systematic review, meta-analysis, and metaregression analysis. Arch Gen Psychiatry. 2006;63:530-8.

47. Yochim BP, MacNeill SE, Lichtenberg PA. "Vascular depression" predicts verbal fluency in older adults. J Clin Exp Neuropsychol. 2006;28:495-508.

48. Forlenza OV. Transtornos depressivos na doença de Alzheimer: diagnóstico e tratamento. Rev Bras Psiquiatr. 2000;22(2):87-95.

49. Canineu PR. Prevalência de demência na população de pacientes idosos internados no serviço de saúde: "Dr. Cândido Ferreira" da prefeitura municipal de Campinas [dissertação]. Campinas: Faculdade de Educação, Universidade Estadual de Campinas; 2001.

50. Carthery-Goulart MT, Areza-Fegyveres R, Schultz RR, Okamoto I, Caramelli P, Bertolucci PHF, et al. Versão brasileira da escala Cornell de depressão em demência (Cornell Depression Scale in Dementia). Arq Neuropsiquiatr. 2007;65:912-5.

51. Teri L, Wagner AW. Assessment of depression in patients with Alzheimer's disease: concordance among informants. Psychol Aging. 1991;6(2):280-5.

52. Peluso MAM, Andrade LHSG. Physical activity and mental health: the association between exercise and mood. Clinics. 2005;1(60):61-70. 